\title{
El impacto de la inserción laboral de personas con discapacidad en las unidades educativas de la ciudad de Ambato
}

\author{
The impact of the labor insertion of people with disabilities in the educational \\ units of the city of Ambato
}

\author{
Patricia Eugenia Villacrés Jínez. ${ }^{1}$, María Luisa Rodríguez Benavidez. ${ }^{2}$ \& Mayra Belén \\ Burbano Ronquillo. ${ }^{3} \&$ Jenny Elizabeth Gaibor Delgado. ${ }^{4}$
}

Recibido: 12-09-2019 / Revisado: 06-10-209 /Aceptado: 07-11-2019/ Publicado: 05-12-2019

\begin{abstract}
DOI: https://doi.org/10.33262/cienciadigital.v3i4.2.1003

The labor insertion of people with disabilities has achieved transcendental spaces in Latin America, especially in the past decade; Regarding the labor indexes, these refer to the issue of human rights in health, education, housing and work, but this aspect is still a great problem. Ecuador has policies that identify these men as the priority care group, in this way the primary objective of the investigation, sees a high commotion because it addresses situations of the positive insertion of disabled people who also obtain a resounding majority of times, being a valuable social segment, identical to the records of labor benefits in the educational units of the city of Ambato. In the work of the functions they encounter obstacles, not because of their condition, but because of how the community is organized. The inconveniences that have to happen to acquire employment, are the rules and regulations restricted and that when guaranteeing their access, do not comply with the stipulations of the legislation; It is notorious in several public and private companies that disabled employees are not treated with equality, stereotypes of marginalization and compassion are created for this human species, reducing their self-esteem because they are considered useless or non-productive. The population was delimited in 1244, the sample is 294 . On the methodology that was used can be mentioned: Methods of Analysis: Theoretical Framework, through the participation of concepts based on the authorship of several specialists; the literature and inherent literary productions; Descriptive Method that meant using the survey technique with its tool called a questionnaire, in order to obtain information that connects, the same that helped inspire parity and accessibility to multiple opportunities and in all citizen areas.

\footnotetext{
${ }^{1}$ Instituto Tecnológico Superior “Luis A. Martínez”. Redes y Telecomunicaciones. Ambato, Ecuador, luisarob15@yahoo.com

2 Instituto Tecnológico Superior "Luis A. Martínez". Redes y Telecomunicaciones. Ambato, Ecuador, mayraburbano1983@yahoo.es

${ }^{3}$ Instituto Tecnológico Superior “Luis A. Martínez". Redes y Telecomunicaciones. Ambato, Ecuador, otapgenio1@ hotmail.com

${ }^{4}$ Universidad de las Fuerzas Armadas Espe - Esforse, Latacunga, Ecuador, jennygaibor@yahoo.com
} 
Keywords: Disability, Equality, Performance, Job, Marginalization, Stereotypes.

\section{RESUMEN}

La inserción laboral de personas con discapacidad, ha logrado espacios transcendentales en América Latina, sobre todo en la década pasada; respecto a los índices laborales éstos referencian el tema de los derechos humanos en salud, educación, vivienda y trabajo, pero este aspecto, aún sigue constituyendo una gran problemática. El Ecuador tiene políticas que identifican a dichos hombres como el grupo de atención prioritaria, de esta manera el objetivo primordial de la investigación, vislumbra una alta conmoción porque aborda situaciones de la inserción positiva de discapacitados quienes también obtienen la mayoría de veces, un rotundo, siendo ellos un valioso segmento social, idéntico que los registros de beneficios laborales en las unidades educativas de la ciudad de Ambato. En el quehacer de las funciones ellos encuentran obstáculos, no debido a su condición, sino por cómo está organizada la colectividad. Los inconvenientes que tienen que pasar para adquirir empleo, son las normas y reglamentaciones restringidas y que, a la hora de garantizar su acceso, no cumplen con lo estipulado por la legislación; es notorio en varias empresas públicas y privadas que los colaboradores inhabilitados no son tratados con igualdad, se crean estereotipos de marginación y compasión destinados a esta especie humana, disminuyendo su autoestima por ser considerados inútiles o no productivos. La población se delimitó en 1244, la muestra es de 294. Sobre la metodología que se utilizó se puede mencionar: Métodos de Análisis: Marco Teórico, a través de la participación de conceptos fundamentados en la autoría de varios especialistas; la bibliografía y producciones literarias inherentes; Método Descriptivo que significó emplear la técnica de la encuesta con su herramienta llamado cuestionario, a fin de obtener información que conecte, la misma que coadyuve a inspirar la paridad y accesibilidad a las múltiples oportunidades y en todos los ámbitos ciudadanos.

Palabras claves: Discapacidad, Igualdad, Rendimiento, Empleo, Marginación, Estereotipos.

\section{INTRODUCCIÓN}

El texto de la Constitución del Ecuador (2008), garantiza el buen vivir de los seres de la humanidad que tienen discapacidad, lo cual está promulgado en su artículo 47, numeral 10, de tal forma que tengan oportunidad de alcanzar los bienes y servicios que presta la sociedad y el Estado; a la par avala su ingreso a las organizaciones y que éste se desarrolle en equidad de condiciones y ventajas, de modo que permita fomentar sus capacidades, con base a lo que desarrollan las políticas públicas, que aprueban su incorporación en entidades públicas y privadas; así en la Ley de Discapacidades Art. 4 Numeral 8. Accesibilidad. Art. 12 de su reglamento, se establece su garantía y el paso a laborar en los diferentes sectores productivos, sea en la urbe o en el sector rural, fomentando un grado superior de autonomía en su diario vivir.

Lo examinado, en lo que se refiere a la afiliación laboral de personas con disminución en sus facultades en las unidades educativas de Ambato, nos permite situar pensamientos e ideas justamente en éste que es un problema aparentemente solucionado, ya que desde que entró en vigencia la Ley Orgánica de Discapacidades, se empezó a otorgarles ciertos patrocinios, pero que en la mayoría de casos son de mínimo cumplimiento, mucho más cuando se trata del asunto conocido como modalidad de colocación ocupacional a estos sujetos en riesgo e insertos en el análisis específico del área educativa, desencadenándose esto en escenarios en que los 
individuos en referencia, pasan a ser parte de los indicadores del subempleo en el país, viéndose obligados a desempeñarse en actividades disímiles a su preparación académica, que les proporciona escasos recursos, con los cuales no pueden asegurar la subsistencia propia y de sus familias; asimismo la falta de excelentes opciones los conduce a emplearse en alternativas que no disponen de los medios favorables de seguridad, implicando esto que deban laborar en entornos frecuentemente precarios, e incluso poniendo en contingencia sus vidas e integridad física, ya que se están dedicando a diligencias no adecuadas. De ahí la importancia de esta búsqueda, pues lo señalado continúa sucediendo, a pesar de que el artículo 53 constitucionalmente afianza el cuidado a los incapacitados, y reconoce el derecho de la gente con diverso porcentaje de minusvalía, por medio de procedimientos alternativos: tales como lengua de señas, oralismo, sistema braille y otras, solo que los establecimientos evitan complicaciones al contratar a personal discapacitado para la ejecución de sus tareas, y seleccionan preferiblemente a quienes no padecen de mucha dificultad para movilizarse, hablar, o ver; es por eso que en ocasiones se observa que las empresas eligen a quienes cuenten con un pequeño índice de deficiencia en cualquiera de sus sentidos, lo que deja entrever que la sección de trabajo social, no cumple acertadamente su función ética, profesional, y social; siendo lo peor el hecho de no acatar la ley enfáticamente y las prebendas que esta agrupación masculina, femenina, niños y niñas tienen, limitándose a cumplir únicamente con lo que estipula la última reforma al Código de Trabajo publicada en el Registro Oficial, Suplemento 167 del 16 de diciembre de 2005, y actualizado a mayo del 2013; estableciéndose allí que el total de la contratación de personas con discapacidades será del $4 \%$ en una institución que cuente con 25 trabajadores, en lo público y privado (Código del Trabajo, 2013).

Siguiendo el artículo de Cazar, las características muy notables que evidencian la condición de los ciudadanos son: Educación que registra el 37.9\% de la localidad con discapacidad y no cuentan con ningún nivel de instrucción; el $42 \%$ ha cursado alguna vez la escuela primaria; el $10.5 \%$ ha estudiado en cierta ocasión la secundaria y apenas el $1.8 \%$ accedió a la universidad. De los datos correspondientes, el 6.1\% asistió a escuelas de enseñanza especial y el $0.5 \%$ de esta población a escuelas con programas de integración. Referente al Empleo, el $18.2 \%$ con inhabilidades trabajan, el $29.1 \%$ están en la desocupación y que no se enfilan para el desempeño de cualquier función, por la categoría de su imposibilidad; la mujer es discriminada en una cantidad considerable para ingresar a trabajar, y es complicado el problema en la zona urbana, más que en la rural. El 31\% de gente ocupadas están laborando en la fuerza agrícola; el 17\% se dedican al comercio, el resto lo efectúan en servicios, industria, manufactura, y labores domésticas, o asignaciones de libre ejercicio y profesionalismo. De los que laboran, el $36.2 \%$ generan entradas económicas, el $84.4 \%$ no gozan de los servicios del IESS y sólo el 7\% de ellos han recibido capacitación operacional (Cazar, Coello, Jaramillo, M., \& Ortiz, 2016).

El Instituto Nacional de Estadísticas y Censos (INEC, 2017), en su informe relacionado a las dificultades encontradas en el proceso de insertar a empleados u obreros imposibilitados en el mundo funcional, dice que existe paralelismo con su conocimiento; y la fuerte interrogante es, si no hay vigilancia educativa apropiada, por medio de qué podrían prepararse para el área laboral? y si logró tener algún saber; del mismo modo se enfrenta con la incertidumbre de ser aceptado o no en especificadas plazas; pese a que consta el régimen que asegura su ingreso. Los vastos esfuerzos desarrollados por colectivos y establecimientos, triunfando en la inclusión, en vacantes destinadas a estos personajes, continúa siendo muy reducida y al ubicarse geográficamente en Ambato, provincia de Tungurahua, señalan que son 7.472 que están registrados en el Consejo Nacional para la Igualdad de Discapacidades (CONADIS) de los 
cuales únicamente el $4 \%$ han ingresado a un cargo seguro en comercio o plantel alguno, esto lo demuestra el Instituto Nacional de Estadísticas y Censos. Dentro de la inspección, en primer lugar, se ubican los inscritos con nulidad física, en tanto que la visual se ubica en el cuarto lugar. En el espacio ya citado, se evidencia una cantidad superior de profesionales activos y las atrofias que poseen en número elevado es la alteración externa; hoy en día en el país cada vez se van implementado estrategias que identifican al mencionado conjunto, dado que son ese colectivo de protección preferente y vulnerable, con el fin de hacer efectivos las potestades que tienen acerca de la cultura, enseñanza, sanidad, rehabilitación, habilitación, admisión y colocaciones. En la provincia de Tungurahua dialogar de la inclusión de humanos imposibilitados se traduce en algo difícil, en virtud de que las organizaciones en ocasiones se convierten en esa pared, que no permiten a la agrupación con incapacidades incorporarse a laborar, porque así se desenvuelvan con poca normalidad en el ámbito familiar, educativo, de salud, y puestos del accionar laboral, significan enormes egresos de dinero para ellos. Sin embargo y pese a que económicamente muchos entes que llevan a cabo prácticas de índole social, entre otros el gobierno estatal, municipales, parroquiales, fundaciones, ONG's, etc., consiguen integrar a estos hermanos, también van alcanzando concienciar a los empresarios e instituciones con el fin de que no cumplan solamente con ese $4 \%$ exigido de acogimiento, sino que la adhesión sea indistinta del tipo de minusvalía que adolecen, pues al percibir esta discriminación, se sienten marginados en su hábitat y financieramente igualmente ligados a procesos de elevada exclusión, forjando ciudadanos con estigmatización y contravenciones limitantes en acceder al aprendizaje, deberes y gestión plena, pudiendo contar con una mejor subsistencia, activa y participativa (INEC, 2017).

De conformidad a las cifras del INEC, en el año 2017, se considera la siguiente información estadística de quienes trabajan en las unidades educativas del cantón Ambato; indagación realizada sobre la base del Consejo Nacional para la igualdad de discapacitados.

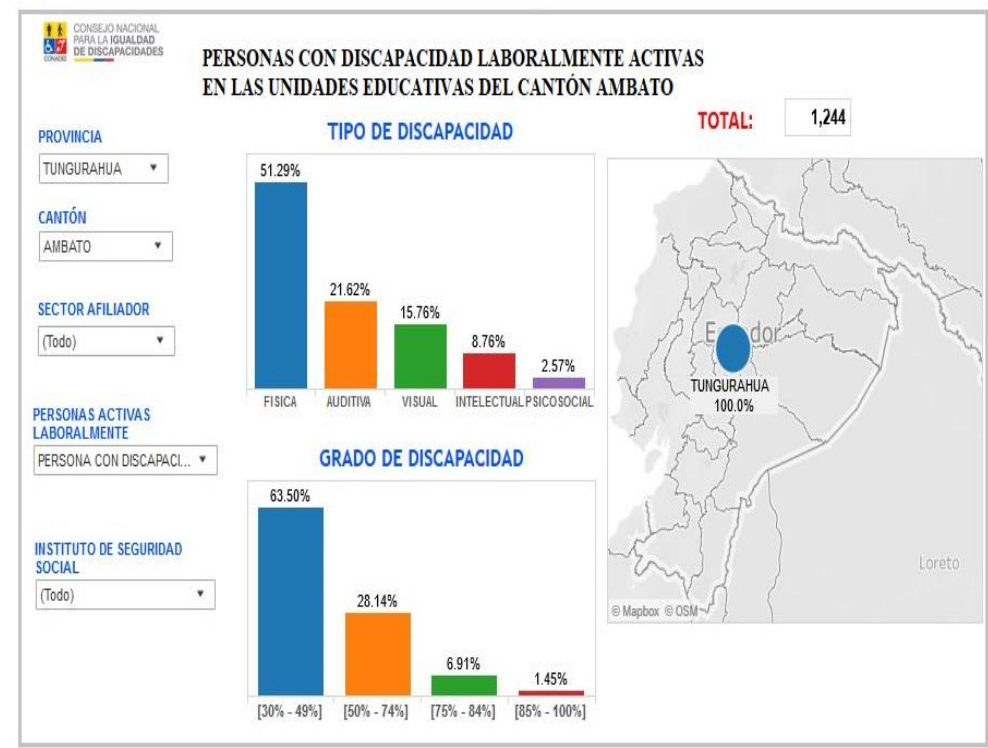

Figura 1: Discapacitados

Fuente: Elaborado por las autoras, datos extraídos del INEC, 2019

En tanto que González (2010) en su reseña en calidad de directora de la Fundación Integralia DKV, menciona los prejuicios que algunas entidades los ubican como obstaculizadores para contratar a señores o señoras con discapacidad y señala que los 
empresarios consideran entre éstos, el ausentismo laboral, el costo de adaptación al cargo, bajo rendimiento, rotación permanente, entre otros. Sin embargo, ella acota que, en la Fundación mencionada anteriormente, ya con casi 20 años trabajando con esta gente, no ha existido ningún inconveniente en continuar contratándolos, porque ellos determinan cuáles son las necesidades y capacidades a fin de que se adapten a la organización. Sugiere al contrario que los negocios y corporaciones, se benefician al dar trabajo a estos individuos, tal vez en pagos de impuestos, óptimo clima organizacional, etc., ya que fomentan el progreso, motivación y compañerismo entre empleados, provocándoles un efecto de pertenencia hacia la organización, y afianza los valores por los que sienten empatía; no obstante, estas realidades no son consideradas pues se detecta la ausencia de interés por tan siquiera conocer a este personal.

\section{METODOLOGÍA}

En este acápite se revisa esta área temática con base al cálculo del tamaño de la muestra, a partir de la población que asciende al número de 1244 y una muestra de 294; el artículo se basa en Métodos de Análisis: Método Descriptivo, donde se aplica la encuesta con su herramienta denominada cuestionario. El propósito es alcanzar testimonios en referencia a la indagación; Marco Teórico: que permite conocer y profundizar de forma general y específica las distintas instancias de la trama que se ha examinado; Método Empírico: sintetizando antecedentes derivados del uso de técnicas: recolección de datos, utilización de instrumentos puesto que el caso requiere historiales en correspondencia al interesante texto que nos ocupa; denotándose que la orientación cualitativa identifica un porcentaje no satisfactorio de seres con discapacidad que son una serie económicamente activa en planteles académicos; dato numérico que debe desplegar una reflexión hacia la sociedad para impulsar y promover la consonancia y asequibilidad al mercado productivo en las numerosas esferas de la colectividad; y la cuantitativa advierte la comprobación de las citas obtenidas instituidas en el manejo de la estadística que logra establecer la eficiencia y seguridad de los testimonios conseguidos, marcando cierto influjo negativo y perjudicial que sufren ante la injusticia de no ser insertados en algún trabajo como un derecho que ostentan. De igual manera estas experiencias contienen notas bibliográficas y documentales, tales son: libros, revistas, sitios web, etc.; esto va paralelo a la exploración en la internet e intranet.

En esta investigación, la localidad propuesta para el estudio es la ciudad de Ambato, con las últimas fichas de los empadronados en elecciones y emitidos por el INEC; el informe del temario en este ámbito data del año 2017; se usó la encuesta con su instrumento destacado como cuestionario, y a continuación se detalla lo correspondiente a la (Tabla 1)

Tabla 1. Población discapacitada

\begin{tabular}{ccc}
\hline$\#$ & \multicolumn{1}{c}{ Miembros } & Técnica/Herramienta \\
\hline $\mathbf{1 2 4 4}$ & $\begin{array}{l}\text { discapacitada que trabaja en las } \\
\text { unidades educativas de Ambato. }\end{array}$ & Encuesta-cuestionario \\
\hline $\begin{array}{l}\text { Elaborado por: } \\
\text { Fuente: }\end{array}$ & $\begin{array}{c}\text { Autoras } \\
\text { INEC, } 2019\end{array}$ & \\
\hline
\end{tabular}

Toda vez que la población de 1244 es considerablemente extensa, se deriva el empleo de la fórmula y se logra una muestra que da lugar a indagar; en esta ocasión no es procedente actuar con ligereza por cuanto si se toma ésta, o la más grande de lo necesario, constituye desperdicio de los recursos, mientras que las muy pequeñas a menudo recaban resultados sin 
uso práctico y nada confiable. Respecto al tamaño de la muestra que es de 294 , de distintas edades y estratos, mayores de edad, se realiza el muestreo probabilístico por conveniencia, ya que es una destreza de muestreo no probabilístico, y no aleatorio utilizada para crearlas de acuerdo a la facilidad de aceptación y ante todo que puedan ser incluidos con la disponibilidad de los habitantes, que son una fracción de ese ítem en un intervalo de tiempo otorgado; se han elegido a los miembros solo por la proximidad y luego conseguir una notificación acerca de los inconvenientes críticos percibidos, sobre las colocaciones en instituciones y los conflictos del personal discapacitado en los centros pedagógicos, académicos, educativos y empresariales.

\section{RESULTADOS Y DISCUSIÓN}

Todo lo aquí desplegado es razonable y corrobora la realidad del objetivo y en concordancia a la inserción laboral de las personas con discapacidades en las unidades educativas de Ambato, exigen a que les den exclusiva atención, ya que conforme se halla en la actualidad, perjudica el desarrollo social, psicológico y económico de los desempleados que se involucran en esta congregación vulnerable.

La relación que demuestra Barrera (2018), indica que a los actores con este tipo de limitaciones se les afecta de diferentes formas, aunque soporten estas situaciones, cualquiera de éstas puede estar ocultas y no ser evidentes fácilmente.

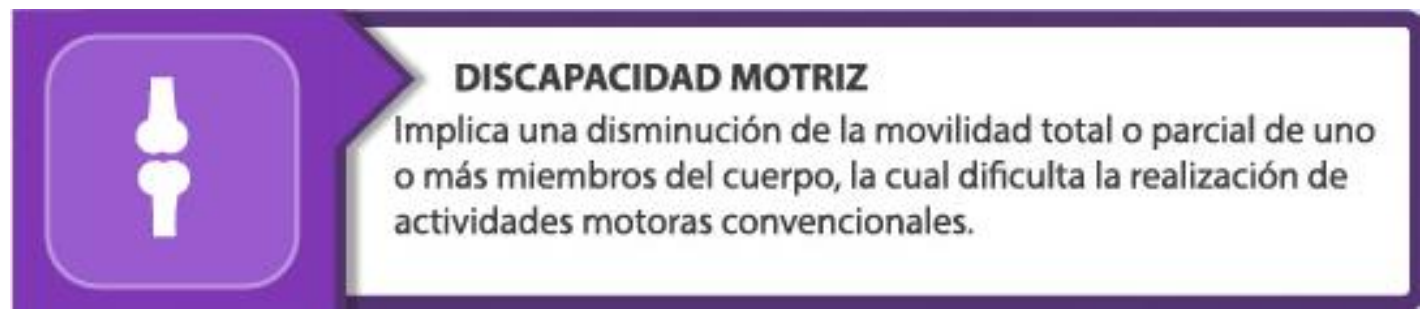

DISCAPACIDAD VISUAL

De acuerdo al grado de limitación de la visión, se suele distinguir entre personas ciegas, que no obtienen información a través del canal visual; y personas con disminución visual, quienes en cambio sí la adquieren mediante dicho canal.

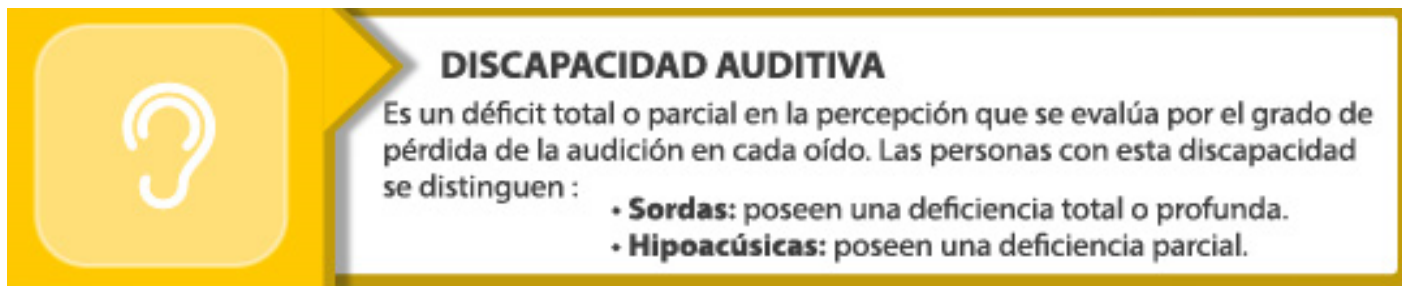

\section{DISCAPACIDAD INTELECTUAL}

Funcionamiento intelectual inferior a la media, limitaciones en áreas de habilidades como la comunicación, habilidades sociales y seguridad, entre otros.
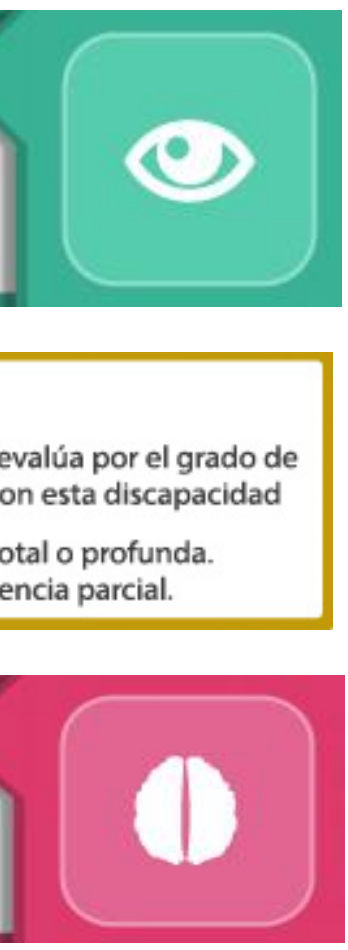


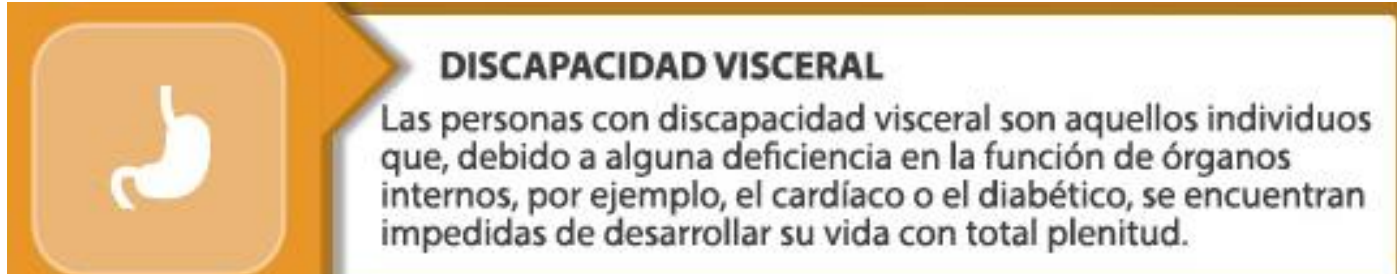

Figura 2: Tipos

Fuente: Elaborado por las autoras, datos extraídos

El sondeo fundamentado en lo que señala Espinoza y Gallegos (2018), en su escrito de la revista Espacios, detecta que aproximadamente mil millones de habitantes en el mundo resisten alguna discapacidad; es decir el $15 \%$ de la población integral, en el que al Ecuador lo consideran como la nación latinoamericana con un creciente indicador de incapacidad, luego va México y finalmente Panamá.

La averiguación efectuada en cuanto a este argumento, arroja los siguientes efectos relativos al caso de que en Ecuador las discapacidades son las que se describen y que el CONADIS igualmente lo confirma. Consta la información en la (Tabla 2)

Tabla 2. Cifras y tipos en Ecuador

\begin{tabular}{ll}
\hline \multicolumn{1}{c}{ SÍNTOMA } & \# \\
\hline Auditivas & 35274 \\
Motriz & 156139 \\
Intelectuales & 69385 \\
Lenguaje & 4661 \\
Psicológico & 12520 \\
Visuales & 35177
\end{tabular}

SUMA: 313336

\section{Elaborado por: Autoras}

Fuente: $\quad$ Investigadora y CONADIS, 2019

Sobre el contexto y un conglomerado formado por hombres y mujeres, la (Figura 2) explica que hay $33.67 \%$ dentro del sexo femenino con discapacidad que se encuentran trabajando muy activamente; y en la sección masculina el 66.33\%; con proliferación de atrofias corpóreas. Cabe mencionar que estos semejantes se hallan entre los 18 a 65 años; aquí el 5,23\% corresponde a adultos mayores de 65 años y que son minusválidos. 


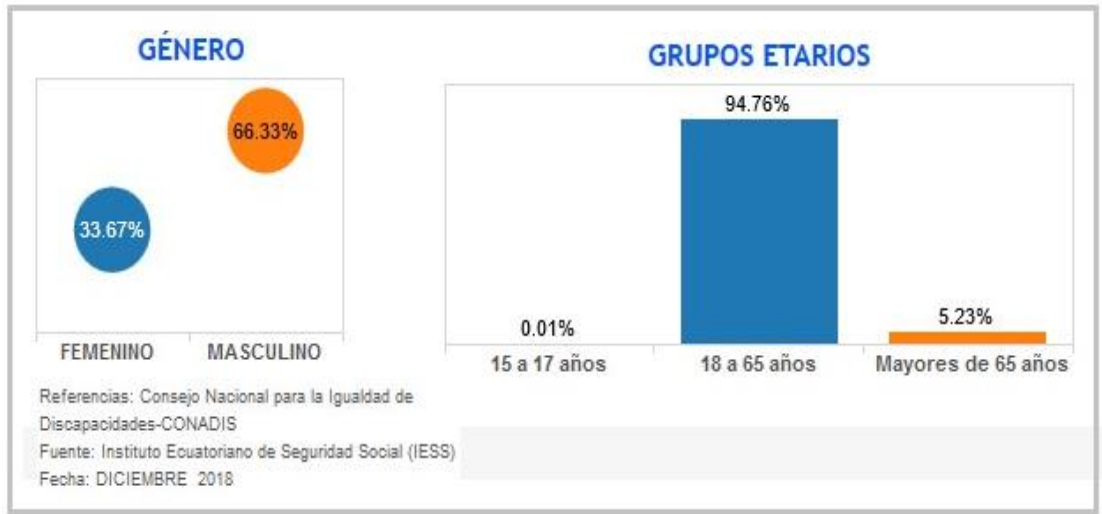

Figura 3: Género y grupos etarios, edad, sexo

Fuente: IESS (2018)

Mientras que la (Tabla 3), fija que en Ambato constan políticas y programas que aportan a los actores discapacitados en el mercado de oficios y tareas, para que consigan trabajo o puedan crear sus propios negocios, emprendimientos o cooperativas en tan solo el $39 \%$.

Tabla 3. Políticas y Programas que aportan a discapacitados

\begin{tabular}{lcc}
\hline \multicolumn{1}{c}{ ALTERNATIVA } & FRECUENCIA & \% \\
\hline SÍ & 89 & $39 \%$ \\
NO & 124 & $47 \%$ \\
NO LO SÉ & 60 & $12 \%$ \\
NO CONTESTO & 21 & $2 \%$ \\
$\quad$ TOTAL & $\mathbf{2 9 4}$ & $\mathbf{1 0 0 \%}$
\end{tabular}

Elaborado por: Autoras

Fuente: $\quad$ Encuesta, 2019

Adicional a la encuesta mencionada anteriormente, con el instrumento se evidenció que aisladamente el $24 \%$ de coterráneos acceden a proyectos ejecutables en las zonas educativas, gremiales y sanitarias que les capaciten con el ánimo de conseguir su plena agregación a la vida cotidiana, tal como consta en la (Tabla 4).

Tabla 4. Proyectos ejecutables en varias áreas

\begin{tabular}{ccc}
\hline ALTERNATIVA & FRECUENCIA & \% \\
\hline Siempre & 70 & $24 \%$ \\
A veces & 210 & $71 \%$ \\
Nunca & 14 & $5 \%$ \\
SUMAN & $\mathbf{2 9 4}$ & $\mathbf{1 0 0 \%}$ \\
\hline
\end{tabular}

$\begin{array}{ll}\text { Elaborado por: } & \text { Autoras } \\ \text { Fuente: } & \text { Encuesta, } 2019\end{array}$

La autora Henriquez \& Edithza (2010), en su argumento manifiesta que por intermedio de investigaciones orientadas se busca eliminar las brechas del desconocimiento que tiene la comunidad; y estos equipos ya comienzan a razonar del valor que otorga la integración de estos amigos que sufren cierta nulidad motora, y se van dando paso firme a las coyunturas que permitan la introducción de ellos al campo laboral y educativo. 
Según el Ministerio del Trabajo, ente gubernamental que fomenta el cumplimiento de obligaciones ocupacionales contenidas en el Código respectivo, efectúa inspecciones con las Direcciones Regionales, Departamento de Seguridad, e Inspectorías, a nivel local y nacional, en coordinación con la Unidad que le compete; esto con el fin de avalar los derechos de los trabajadores con salud normal y de los que también resisten alguna clase de disfunción, verificando que estén inmersos en los negocios, instituciones, etc., acorde a lo que la ley indica; trámites que les permite confirmar además, el acatamiento de disposiciones, como el hecho de que cuenten con edificios estructuralmente construidos con rampas, escaleras apropiadas, ascensores, eliminación de las llamadas "barreras arquitectónicas", computadores y mouse adaptados, y atriles; todo ello lleva a preguntarnos cuánto sabemos de los hermanos con discapacidades ?; tal vez qué es un bastón guía, implante coclear, la andadera, prótesis, muletas, códigos elaborados, lenguaje de señas, etc.?, para ir alcanzando esa propicia comunicación con ellos.

Esta situación objeto de observación, considerada todavía como ese mayúsculo limitante dirigido a esta corporación común, requiere de resoluciones urgentes, donde las carteras gubernamentales deberían normalizar óptimamente los procedimientos, el argumento legal y económico; esto dentro de las posiciones externas y en relación a los internos, las oficinas del sector público y las privadas han de gestionar con el método efectivo el talento humano, clima organizacional, relaciones interpersonales, y estabilidad, con la finalidad de eliminar esa grieta permanente, para que cuando llegue un empleado así, éste sea acogido debidamente, tomando en cuenta sus aprendizajes, habilidades y destrezas, y que su autoestima y estimación se conviertan en el apoyo y alternativas de fortaleza que necesitará en el momento de adaptarse, y alcanzar ganancias de conciliación con el ambiente en el que vivimos, consiguiendo con tal procedimiento palear y/o regularizar los grados de afectación a que están abocados, ya que dar valía a sus atribuciones fija la utopía que se va corrigiendo paulatinamente, puesto que la falla es lograr entender que ser figuras heterogéneas, no significa estar deficientes o tener menos valor que otros.

Por eso la visión que excluye, debe convertirse por el contrario en inclusiva por parte de la comunidad, admitiendo, reconociendo y apreciando esas diferencias de hombres y agrupaciones, visto que son esa expresión variada del capital humano. Deriva de allí la creación de sitios de participación, asegurando a todos que la tendencia es hacia el óptimo desarrollo de sus facultades físicas, orgánicas, comunicativas, éticas, intelectuales y emocionales; se trata en sí de habilitar a los miles de discapacitados en actividades ingeniosas, productivas y creativas que beneficien al país, a sus familias y por ende a ellos en especial.

\section{CONCLUSIONES}

Mediante el contenido del documento, revelamos que los representantes de la discapacidad necesitan ser integrados al campo de labores, tendiendo a reestablecer su calidad de vida y sentirse dignificados ya que son hijos de Dios, y debido a que el universo de la producción es importante en la existencia de los individuos sin distinción de ninguna índole.

Esto da la razón y en Ambato que es el lugar de la averiguación, la comunidad, y entidades en general están comprendiendo el dolor por el que pasan los incapacitados, ya que se determinó que no se administra, ni se controla adecuadamente la introducción de estos prójimos a las compañías, para así evitar la discriminación y el menoscabo de sus primacías; asimismo con el apoyo y compromiso de amigos y familiares que sustenten sus aspiraciones, consiguiendo involucrarse en el mantenimiento y mejora continua, apoyándoles en lo que 
precisan, apartando los miedos que naturalmente surgen en ciertos casos, y eliminando en otros, unas cuantas circunstancias egoístas al no merecer esa inclusión en espacios de trabajo, apoyando la supervivencia de aquellos que lo necesitan; conociendo los requerimientos y expectativas de éstos.

La verdad es que a estas personas, al optar por una responsabilidad, ciertamente se les torna más difícil, es por eso que autoridades, organismos, familiares, y amigos tienen que propender a la adaptación en desempeños alternativos: teletrabajo, ocupaciones guiadas o con ayuda, acciones adaptadas en los múltiples sitios, etc., sin que ellas lleguen a ser excesivas pues el emplear mucha diferenciación con ellos, siempre es contraproducente y se torna negativo en la efectiva agregación de los elementos sociales de quienes estamos hablando.

Finalmente, la concienciación completa y con la noble intención de que exista esa real unificación profesional y humana, se expresa en la circunstancia de que, a los distintos tipos comunitarios, políticos y económicos, les corresponde aportar con recursos materiales, financieros y capital humano que contribuyan a plasmar este logro en beneficio de los más necesitados.

\section{REFERENCIAS BIBLIOGRAFÍCAS}

Barrera, J. H. (14 de agosto de 2018). Tipos de discapacidad. Recuperado el 8 de junio de 2019, de https://www.incluyeme.com/wpcontent/uploads/2015/07/Infograf\%C2\%B0a_03_b.jpg

Barrera, J. H. (14 de agosto de 2018). Tipos de discapacidades. Recuperado el 8 de octubre de 2019, de https://www.incluyente.com/wpcontent/uploads/2015/07/infograf\%C2\%B0a_03_b.jpg

Cascant, N. P. (2014). Investigación Científica (Segunda ed., Vol. III). (Noriega, Ed.) México D.F.: McGraw-Hill. Recuperado el 10 de junio de 2019

Cazar, O. (2016). Situación de las personas con discapacidad. Quito-Ecuador: INEC. Recuperado el 10 de junio de 2019, de cienciadigital.org/revistacienciadigital2/index.php/CienciaDigital/article/view/.../788

Cazar, R., Coello, F., Jaramillo, A., M., M., \& Ortiz, C. (2016). Universidad y Discapacidad: la discapacidad en la Universidad Central del Ecuador. Revista Ciencias Sociales. Revista Académica Indexada de la Facultad de Ciencias Sociales y Humanas del Ecuador., 10. Recuperado el 26 de octubre de 2019, de http://revistadigital.uce.ec/index/vieuv/1232.

Código del Trabajo. (2013). En L. C. CODIFICACIÓN (Ed.). Quito, Pichincha, Ecuador: Editora Nacional. Recuperado el 25 de octubre de 2019, de http://www.trabajo.gob.ec/wp-content/uploads/2015/03/CODIGO-DEL-TRABAJO1.pdf

CONADIS. (2015). cifra de discapacidad en el Ecuador. Quito -Ecuador. Recuperado el 10 de junio de 2019, de https://www.consejodiscapacidades.gob.ec/ 
Constitución - Ecuador. (2008). Constitución de la República del Ecuador. En A. Nacional, Constitución de la República del Ecuador (pág. 218). Quito, Pichincha, Ecuador:

Editora Nacional. Recuperado el 22 de octubre de 2019, de https://www.oas.org/juridico/mla/sp/ecu/sp_ecu-int-text-const.pdf

Constitución de la República del Ecuador. (2008). Garantías al Buen Vivir. Quito-Guayaquil: Ministerio del Gobierno. Recuperado el 10 de junio de 2019, de https://www.oas.org/juridico/mla/sp/ecu/sp_ecu-int-text-const.pdf

Convención de la UNE. (2014). Discapacidad. Quito-Ecuador: ONU. Recuperado el 9 de mayo de 2019, de https://www.incluyeme.com/wpcontent/uploads/2015/07/Infograf\%C2\%B0a_03_b.jpg

Espinoza, M., \& Gallegos, D. (22 de 12 de 2018). Inserción Laboral de las personas con discapacidad en Ecuador. Revista Espacios, 39(51). Recuperado el 12 de octubre de 2019, de https://www.revistaespacios.como/a18v39n51/a18v39n51p03.pdf

González, C. (2010). https://dkvintegralia.org. (C. S.L., Ed.) Recuperado el 7 de octubre de 2019, de https:www.dkvintegralia.org/por-que-las-empresas-no-contratan-a-personascon-discapacidad/

Henriquez, E. (2010). Inclusión o integración de las personas con discapacidad. Recuperado el 12 de septiembre de 2019, de monografias.com/trabajos82/inclusion-integracion-depersonas-discapacidad 3. shtml

Herrera, L., Medina, F. A., \& Naranjo, L. G. (2018). Tutoría de la Investigación Científica (Cuarta ed., Vol. III). (H. M. Naranjo, Ed.) Ambato-Ecuador, Tungurahua, Ecuador: Corona S.A. Recuperado el 10 de junio de 2019

IESS. (2018). Consejo Nacional para la Igualdad de Discapacidades. Quito-Ecuador: CONADIS. Recuperado el 10 de junio de 2019, de https://www.consejodiscapacidades.gob.ec/estadisticas-de-discapacidad/

INEC. (2017). Informe sobre Discapacidad en la ciudad de Ambato. Instituto Nacional Ecuatoriano de Estadísticas y Censos, Quito-Ecuador. Recuperado el 27 de octubre de 2019, de https://www.consejodiscapacidades.gob.ec/estadisticas-de-discapacidad/

INEC. (2017). Informe sobre Discapacidad en la ciudad de Ambato. Instituto Nacional de Estadísticas y Censos, Quito. Recuperado el 27 de octubre de 2019, de https://www.consejodiscapacidades.gob.ec/estadisticas-de-discapacidad/

INEC. (2019). Población por provincias, cantones y parroquias del Ecuador, año 2018. Ambato-Ecuador: INEC.

Ley de Discapacidades. (2015). Art.14. Numeral 8. Accesibilidad, Art. 12. Quito-Ecuador. Obtenido de obi.itb.edu.ec/public/docs/ley_organica_disccapacidades_ecuador2.pdf

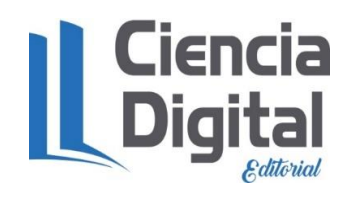




\section{PARA CITAR EL ARTÍCULO INDEXADO.}

Villacrés Jínez, P., Rodríguez Benavidez, M., Burbano Ronquillo, M., \& Gaibor Delgado, J. (2019). El impacto de la inserción laboral de personas con discapacidad en las unidades educativas de la ciudad de Ambato. Ciencia Digital, 3(4.2), 43 - 54. https://doi.org/10.33262/cienciadigital.v3i4.2.1003

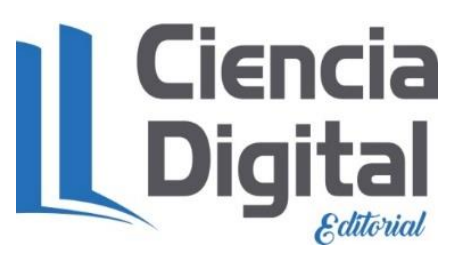

El artículo que se publica es de exclusiva responsabilidad de los autores y no necesariamente reflejan el pensamiento de la Revista Ciencia Digital.

El artículo queda en propiedad de la revista y, por tanto, su publicación parcial y/o total en otro medio tiene que ser autorizado por el director de la Revista Ciencia Digital.
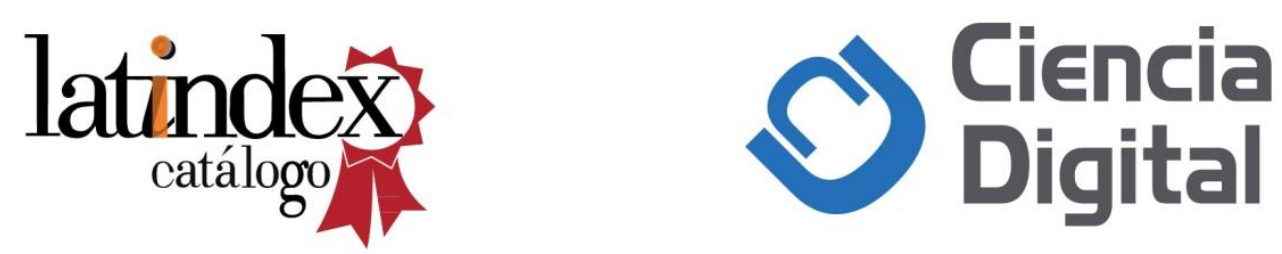